\title{
LRRC41 is a differentially expressed gene in brain metastatic human breast cancer.
}

Shahan Mamoor, MS ${ }^{1}$

${ }^{1}$ shahanmamoor@gmail.com

Metastasis to the brain is a clinical problem in patients with breast cancer ${ }^{1-3}$. We mined published microarray data ${ }^{4,5}$ to compare primary and metastatic tumor transcriptomes for the discovery of genes associated with brain metastasis in humans with metastatic breast cancer. We found that leucine-rich repeat-containing 41, encoded by LRRC41, was among the genes whose expression was most different in the brain metastases of patients with metastatic breast cancer as compared to primary tumors of the breast. LRRC41 mRNA was present at increased quantities in brain metastatic tissues as compared to primary tumors of the breast. Importantly, expression of LRRC41 in primary tumors was significantly correlated with patient recurrence-free survival. Modulation of LRRC41 expression may be relevant to the biology by which tumor cells metastasize from the breast to the brain in humans with metastatic breast cancer.

Keywords: breast cancer, metastasis, brain metastases, central nervous system metastases, leucine-rich repeat-containing 41, LRRC41, systems biology of breast cancer, targeted therapeutics in breast cancer. 
One report described a $34 \%$ incidence of central nervous system metastases in patients compared administration of either neratinib or trastuzumab in conjunction with paclitaxel demonstrated that in a randomized, controlled setting, in breast cancer patients treated with neratinib, not only was the incidence of central nervous system recurrence significantly lower, the time to central nervous system metastasis was significantly delayed as compared to patients administered trastuzumab ${ }^{6}$. The alarmingly high rate of central nervous system metastasis described, as well as data, both anecdotal ${ }^{2}$ and from a randomized, controlled setting ${ }^{6}$ illustrating that treatment with trastuzumab may be associated with these events demands an enhanced understanding of the transcriptional makeup of brain metastatic tissues to support identification of therapeutic targets, whether they are treatment-related or not. We performed a global comparative analysis of primary and metastatic tumors in patients with brain metastatic breast cancer ${ }^{4,5}$. We discovered differential and increased expression of the gene encoding leucine-rich repeat-containing 41, LRRC41, in brain metastatic tissues of patients with metastatic breast cancer.

\section{$\underline{\text { Methods }}$}

We used datasets GSE $10893^{4}$ and GSE $72653^{5}$ for this global differential gene expression analysis of brain metastatic breast cancer in conjunction with GEO2R. GSE10893 was generated using Agilent-011521 Human 1A Microarray G4110A technology with $n=11$ primary breast tumors and $n=3$ brain metastases from patients with breast cancer; analysis was performed using platform GPL885. GSE72653 was generated using Agilent-014850 Whole Human Genome Microarray $4 \mathrm{x} 44 \mathrm{~K}$ G4112F with $n=9$ normal epithelium and $n=9$ tumors of the breast from patients with breast cancer; analysis was performed using platform GPL6480. The Benjamini and Hochberg method of $p$-value adjustment was used for ranking of differential expression but raw $p$-values were used to assess statistical significance of global differential expression.

Log-transformation of data was auto-detected, and the NCBI generated category of platform annotation was used. A statistical test was performed to evaluate whether LRRC41 gene expression was significantly different between primary tumors of the breast and brain metastases in humans with breast cancer using a two-tailed t-test. For Kaplan-Meier survival analysis, we used the Kaplan-Meier plotter online tool ${ }^{7}$ for correlation of LRRC41 mRNA expression levels with recurrence-free survival in $n=3951$ breast cancer patients; auto cut-off was selected.

\section{$\underline{\text { Results }}$}

We performed global comparative transcriptome analysis of primary and metastatic tumor tissues of patients with breast cancer and metastatic breast cancer using published microarray data ${ }^{4,5}$ to describe the transcriptional landscape of brain metastasis in human breast cancer in an unbiased fashion and for the discovery of novel therapeutic targets.

\section{LRRC41 is differentially expressed in the brain metastases of patients with brain metastatic breast cancer.}

Through blind, systems-level analysis of published microarray data ${ }^{4}$, we identified leucine-rich repeat-containing 41, encoded by LRRC41, as a differentially expressed gene in the breast metastatic tissues of humans with breast cancer (Table 1). When sorting each of the genes 
expressed in brain metastases based on significance of difference as compared to primary tumors of the breast in patients with breast cancer, LRRC41 ranked 231 out of 17418 total transcripts (Chart 1), equating to $98.7 \%$ differential expression. Differential expression of LRRC41 in the brain metastases of patients with metastatic breast cancer was statistically significant (Chart 1; $p=6.80 \mathrm{E}-04)$.

To attempt to validate differential transcriptome-wide differential expression of LRRC41 in human breast cancer, we queried a second microarray datase $t^{5}$, here comparing normal breast tissues and primary tumors of the breast. Again, we identified LRRC41 as a differentially expressed gene in the tumor tissues of patients with breast cancer (Chart 2). When sorting each of the genes expressed in primary tumors of the breast based on significance of difference as compared to normal breast tissues, LRRC41 ranked 8706 out of 41093 total transcripts (Chart 2), tumors of patients with breast cancer was statistically significant (Chart $2 ; p=3.61 \mathrm{E}-02$ ). Thus, differential expression of LRRC41, transcriptome-wide, in the primary tumors of women with breast cancer was conserved across two independent microarray datasets, both in primary and metastatic tumor tissues.

\section{LRRC41 is expressed at higher levels in the brain metastases of patients with metastatic breast cancer.}

We obtained exact mRNA expression levels for LRRC41, in primary tumors of the breast and in brain metastasis of patients with brain metastatic breast cancer to determine direction and statistical significance of change in LRRC41 expression in brain metastatic tissues. We observed significantly higher expression of LRRC41 in the brain metastases of patients with breast cancer as compared to primary tumors of the breast: LRRC41 was expressed at $0.13 \pm 0.25$ arbitrary units (AU) in primary tumors of the breast, while it was expressed at $0.84 \pm 0.25 \mathrm{AU}$ in brain metastatic tissues (Figure 1). The difference in LRRC41 mRNA levels between primary tumors of the breast and brain metastatic tissues was statistically significant (Figure $1 ; p=0.000352$ ).

\section{LRRC41 expression is correlated with survival outcomes in human breast cancer.}

We performed Kaplan-Meier survival analysis ${ }^{8}$ in $n=3951$ breast cancer patients in total, to evaluate whether LRRC41 tumor expression was correlated with survival outcomes in breast cancer. We observed a statistically significant correlation between primary tumor expression of LRRC41 and recurrence-free survival (DMFS) in patients with breast cancer (Figure 2). Patients whose primary tumors expressed low levels of LRRC41 possessed median DMFS of 52 months, while patients whose tumors expressed high levels of LRRC41 possessed median RFS of 39 months. This difference in DMFS based on LRRC41 tumor expression in patients with breast cancer was statistically significant, but the false discovery rate (FDR) was high (Figure 2, Chart 3; logrank $p$-value: 0.0076; hazard ratio: 1.18 (1.05-1.34); FDR: $>50 \%$ ).

Thus, by mining published microarray data ${ }^{4,5}$ in an unbiased and systematic fashion, we identified leucine-rich repeat-containing 41, encoded by LRRC41, as among the genes whose expression was most different, transcriptome-wide, in the brain metastases of 
patients with breast cancer, both when compared to primary tumors of the breast and to normal breast tissues; we observed significantly increased expression of LRRC41 in brain metastases as compared to primary tumors of the breast. Further, we found a significant correlation between LRRC41 expression and patient survival outcomes, as recurrence-free survival was significantly greater in patients whose primary tumors expressed higher levels of LRRC41 as compared to patients whose primary tumors expressed lower levels of LRRC41.

\section{Discussion}

We provided evidence here that leucine-rich repeat-containing 41, encoded by LRRC41, is among the genes whose expression is most different in the brain metastases and primary tumors of humans with breast cancer, that LRRC41 mRNA is present at significantly increased quantities in brain metastatic tissues as compared to primary tumors of the breast, and that primary tumor LRRC41 expression is significantly correlated with patient survival outcomes in human breast cancer. Evaluation of the effects of genetic depletion of LRRC41 in mouse models of metastatic breast cancer on metastasis to the central nervous system is merited. Modulation of LRRC41 expression may be relevant to the processes by which breast cancer cells exit the breast, enter the vasculature and/or lymphatics, reside in the lymph nodes, evade immune clearance, breach the blood-brain barrier and colonize the brain. 


\section{References}

1. Lin, N.U., Amiri-Kordestani, L., Palmieri, D., Liewehr, D.J. and Steeg, P.S., 2013. CNS metastases in breast cancer: old challenge, new frontiers.

2. Bendell, J.C., Domchek, S.M., Burstein, H.J., Harris, L., Younger, J., Kuter, I., Bunnell, C., Rue, M., Gelman, R. and Winer, E., 2003. Central nervous system metastases in women who receive trastuzumab-based therapy for metastatic breast carcinoma. Cancer, 97(12), pp.2972-2977.

3. Tsukada, Y., Fouad, A., Pickren, J.W. and Lane, W.W., 1983. Central nervous system metastasis from breast carcinoma autopsy study. Cancer, 52(12), pp.2349-2354.

4. Weigman, V.J., Chao, H.H., Shabalin, A.A., He, X., Parker, J.S., Nordgard, S.H., Grushko, T., Huo, D., Nwachukwu, C., Nobel, A. and Kristensen, V.N., 2012. Basal-like Breast cancer DNA copy number losses identify genes involved in genomic instability, response to therapy, and patient survival. Breast cancer research and treatment, 133(3), pp.865-880.

5. Salhia, B., Kiefer, J., ROSs, J.T., Metapally, R., Martinez, R.A., Johnson, K.N., DiPerna, D.M., Paquette, K.M., Jung, S., Nasser, S. and Wallstrom, G., 2014. Integrated genomic and epigenomic analysis of breast cancer brain metastasis. Plos one, 9(1), p.e85448.

6. Awada, A., Colomer, R., Inoue, K., Bondarenko, I., Badwe, R.A., Demetriou, G., Lee, S.C., Mehta, A.O., Kim, S.B., Bachelot, T. and Goswami, C., 2016. Neratinib plus paclitaxel vs trastuzumab plus paclitaxel in previously untreated metastatic ERBB2-positive breast cancer: the NEfERT-T randomized clinical trial. JAMA oncology, 2(12), pp.1557-1564.

7. Györffy, B., Lanczky, A., Eklund, A.C., Denkert, C., Budczies, J., Li, Q. and Szallasi, Z., 2010. An online survival analysis tool to rapidly assess the effect of 22,277 genes on breast cancer prognosis using microarray data of 1,809 patients. Breast cancer research and treatment, 123(3), pp.725-731. 
Rank: 231

2 Probe ID: 13723

p-value: $6.80 \mathrm{E}-04$

$3 \quad \underline{t}:-4.21$

4 B: -0.236429

Gene: LRRC41

$5 \quad$ Gene name: leucine-rich repeat-containing 41

6

Chart 1: LRRC41 is differentially expressed in brain metastatic breast cancer when comparing brain metastases to primary tumors of the breast.

The rank of global differential expression, the probe/transcript ID, the $p$-value with respect to differential expression transcriptome-wide, $t$, a moderated $t$-statistic, $B$, the log-odds of differential expression between the groups compared, the gene and gene name are listed in this chart. 


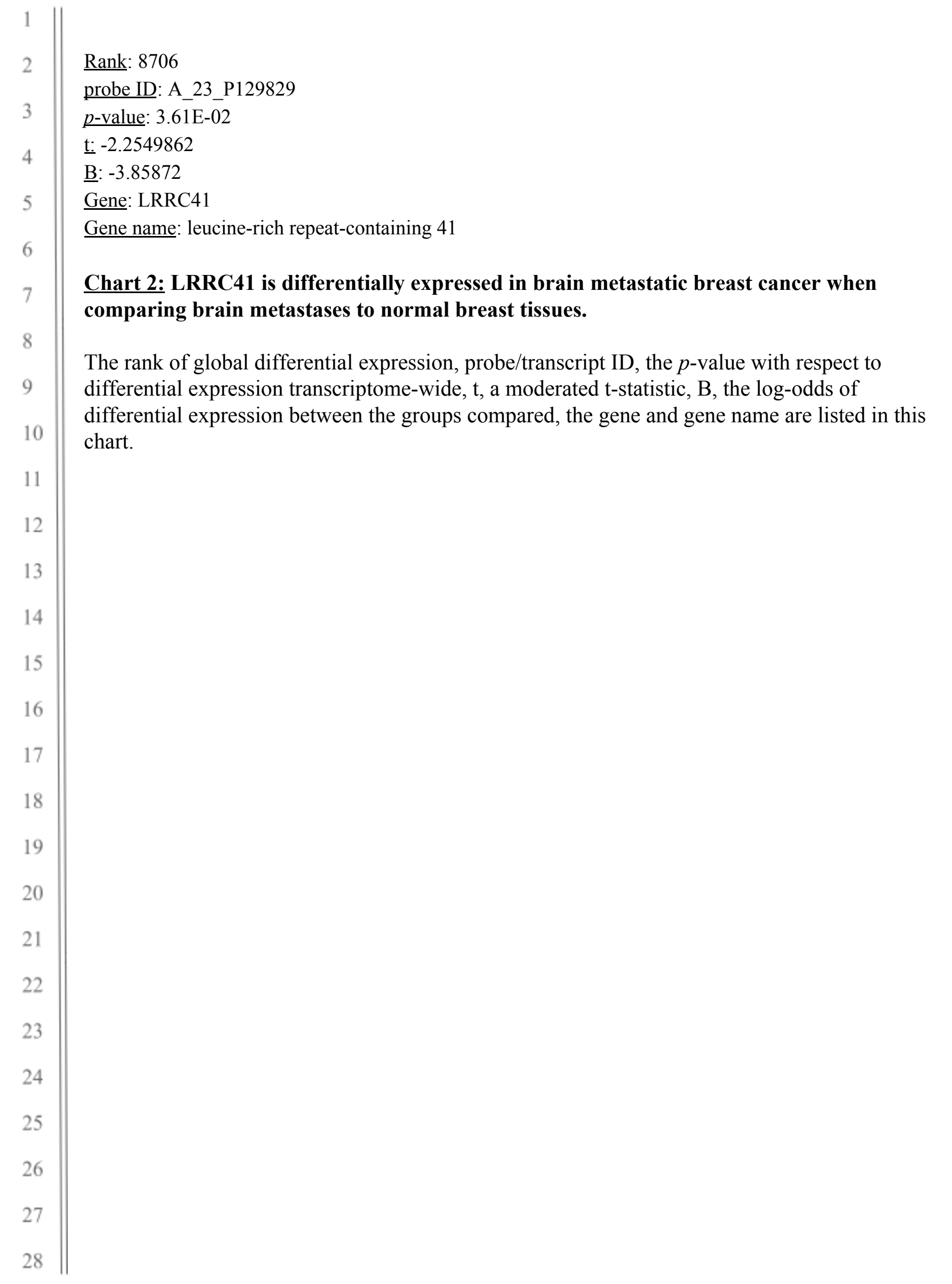




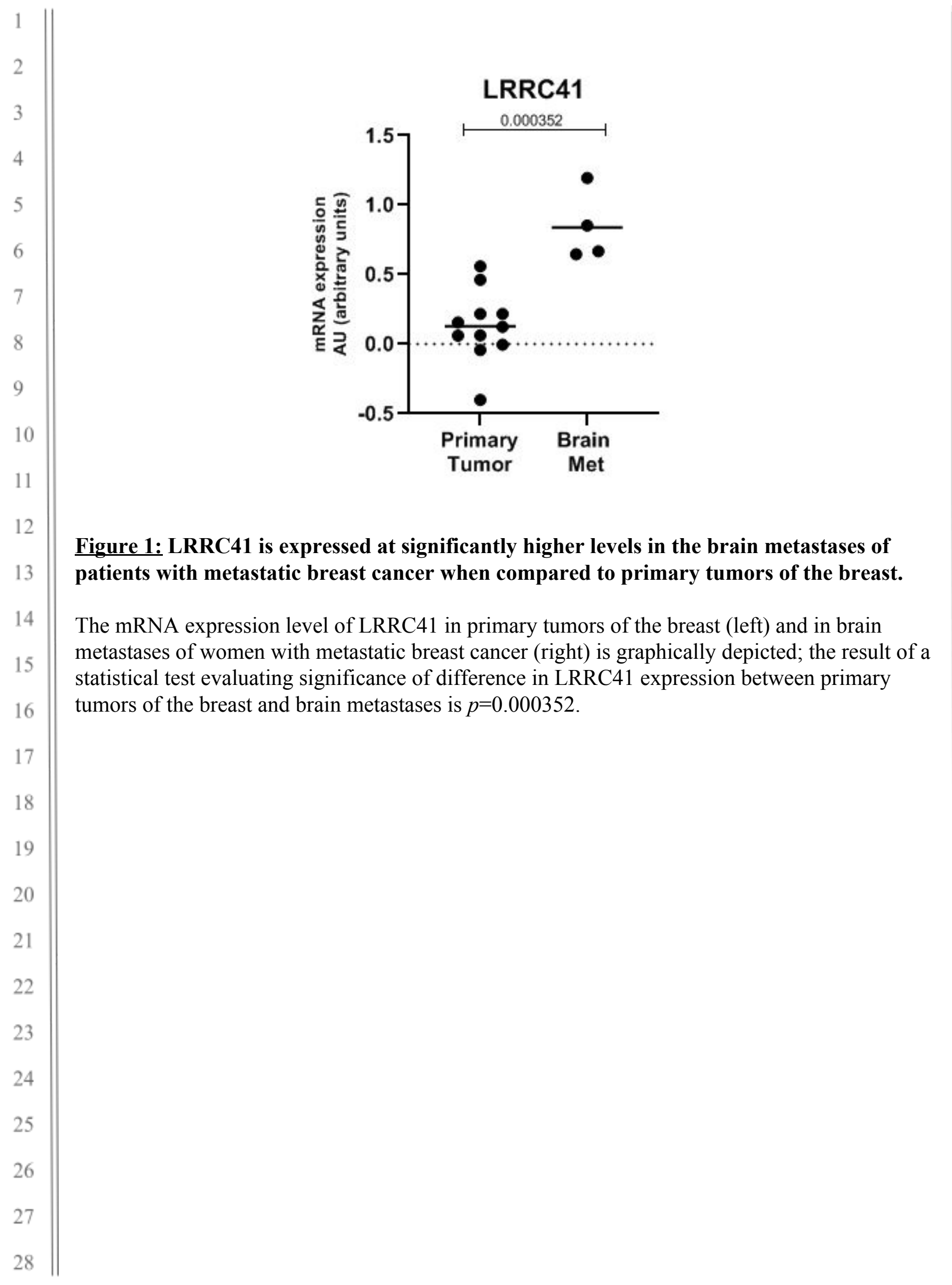




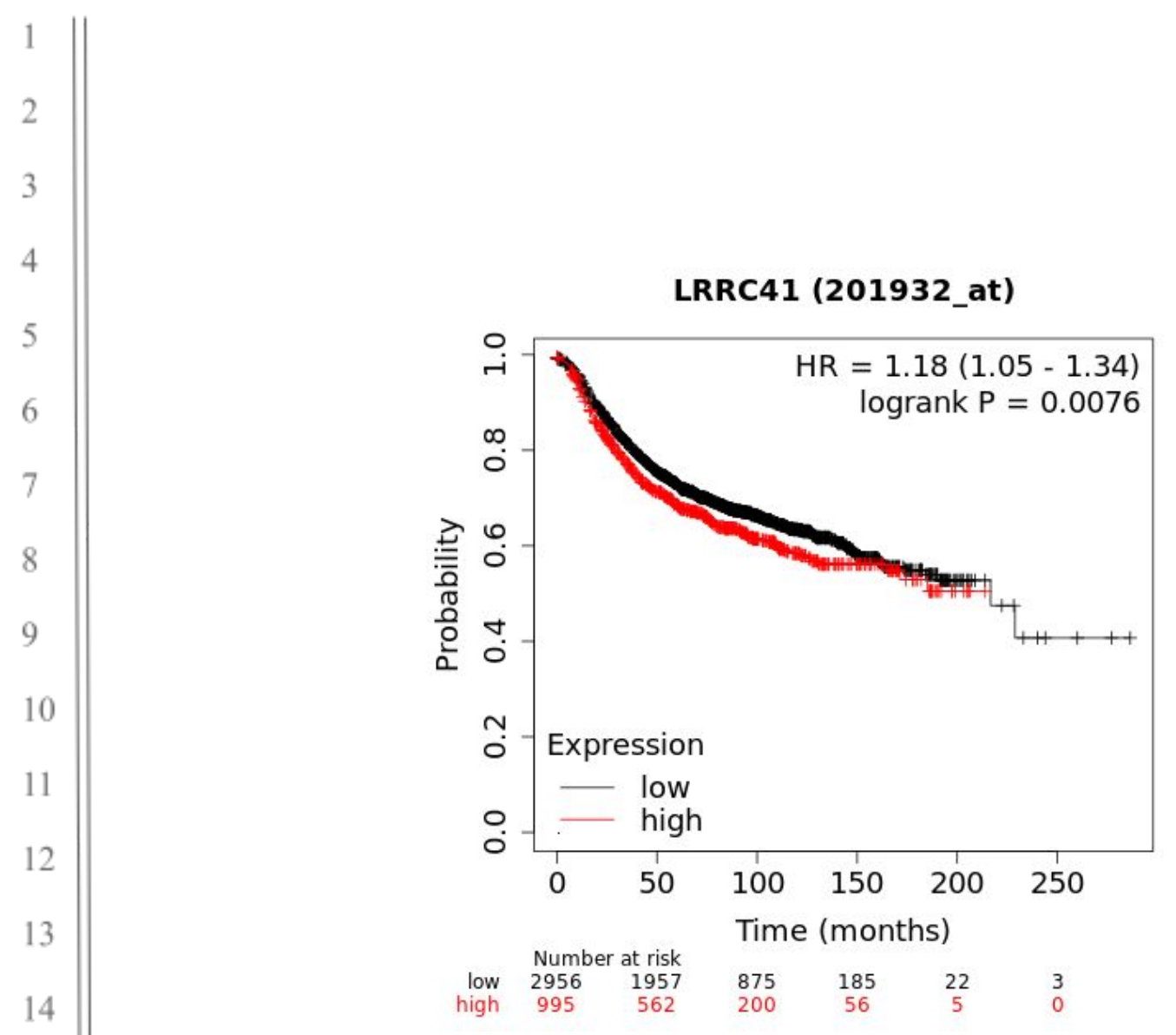

Figure 2: Correlation between LRRC41 primary tumor expression and recurrence-free survival in patients with breast cancer, but with high false discovery rate.

Depicted in this Kaplan-Meier plot is the probability of recurrence-free survival for $n=3951$ total patients stratified into two groups, based on low or high expression of LRRC41 in patient primary tumors. The log rank $p$-value denoting statistical significance of difference in recurrence-free survival when comparing the two groups, as well as hazard ratio for this comparison is listed above. Listed below is the number of patients at risk (number of patients alive) per interval, after stratification based on LRRC41 expression; in the first interval, number at risk is number of patients alive; in each subsequent interval, number at risk is the number at risk less those who have expired or are censored; the false discovery rate is $>50 \%$. 


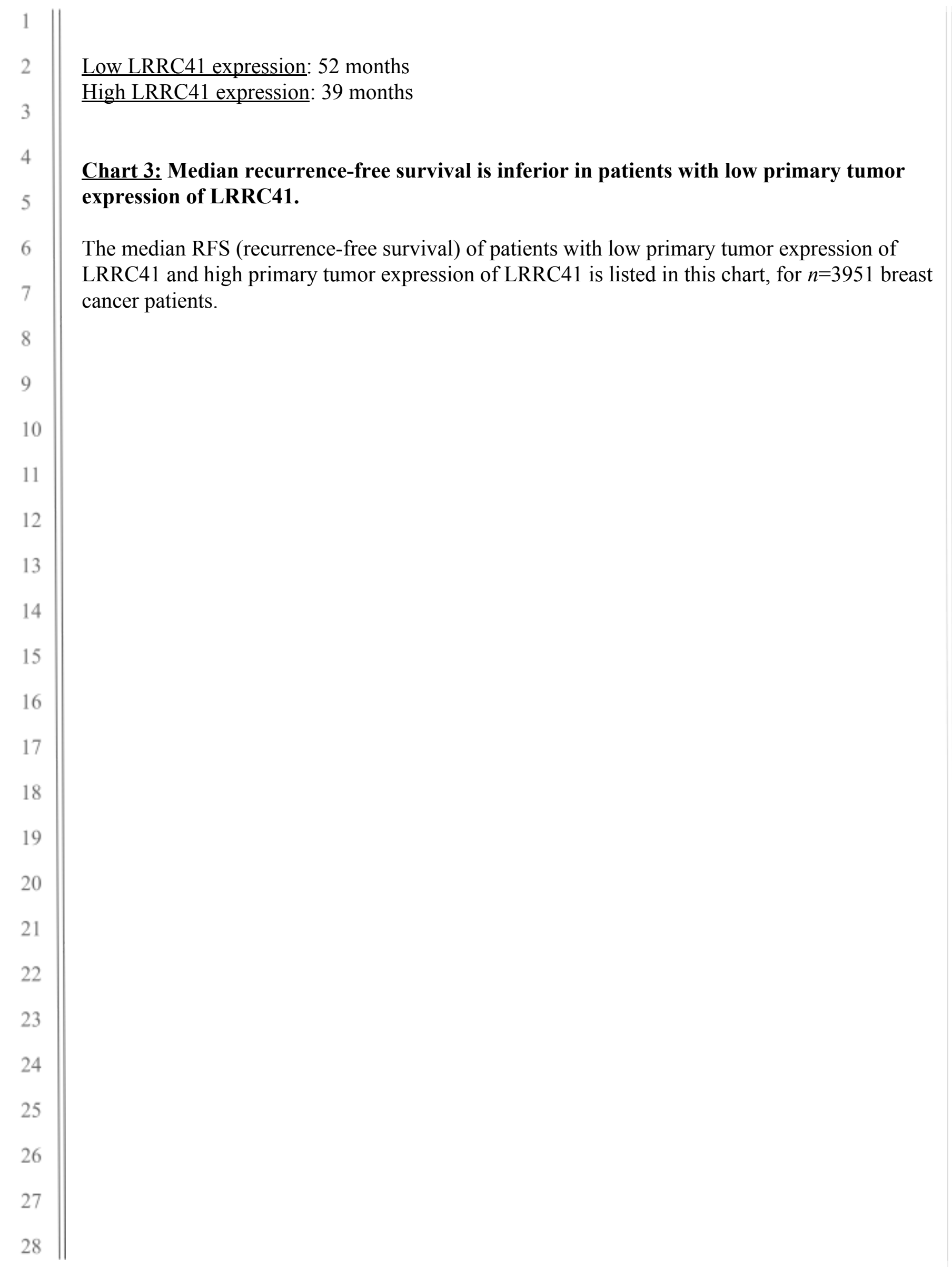

